\title{
SISTEMATIZACIÓN EN UNA REFORMA CURRICULAR
}

\author{
José L. Perea R.*
}

\section{RESUMEN}

La temática tiene como propósito abordar la relación entre el currículo de la formación profesional de la carrera del Psicólogo y el cambio organizacional, en una prospectiva de modernidad del siglo XXI a partir del término de su primer lustro en el ámbito de la educación superior universitaria. También trata la interrelación y consistencia entre las concepciones básicas o nuevas de acuerdo a la finalidad del campo profesional, en la etapa fundamental del diseño curricular, que servirá de soporte en la sistematización de la gestión y evaluación de la estructura curricular. Asimismo, se propicia a que exista la consistencia entre las propuestas de currículo para una formación integral o una formación tecnológica, del ejercicio profesional enmarcado en su normatividad, y de su pertinencia con las demandas de la realidad social, económica y política del país.

Palabras claves: Cambio organizacional, currículo, salud, sistema curricular, sistematización.

\begin{abstract}
The thematic one must like intention to approach the relation between currículo of the professional formation of the race of the Psychologist and the organizacional change, in a prospective of modernity of century XXI from the term of its first lustrum in the scope of the university superior education. Also it deals with the interrelation and consistency between new the basic conceptions or according to the purpose the professional field, in the fundamental stage of the curricular design, that will serve as support in the systematization of the management and evaluation of the curricular structure. Also, it is caused to that the consistency between the proposals of currículo for an integral formation or a technological formation, of the framed professional exercise in its normatividad, and of its pertinencia with the demands of the social reality exists, economic and political of the country.
\end{abstract}

Keywords: Organizacional change, currículo, health, curricular system, systematization.

* Psicólogo colegiado con maestría en Administración de la educación, conciliador extrajudicial, Educador, docente universitario en la UNMSM y universidades privadas. 
Revista de Investigación en Psicología - Vol. 8, N. ${ }^{\circ} 2$

\section{INTRODUCCIÓN}

"..el cambio organizacional se describe como "cambio profundo"

que combina modificaciones internas de los valores de la gente, sus aspiraciones y conductas, con "variaciones externas" en procesos, estrategias, prácticas y sistemas.

En el cambio profundo hay aprendizaje.

No basta con cambiar estrategias, estructuras y sistemas,

También tienen que cambiar las maneras de pensar..."

Peter Senge, La Danza del Cambio.

El contenido del presente trabajo fue expuesto el día domingo 30 de Octubre del 2005, como ponencia en el Congreso Internacional de Psicología, habiendo fallecido el gran maestro Doctor Walter Peñaloza Ramella, reconocido internacionalmente en la comunidad universitaria como el sabio peruano y Amauta de América, impulsor de la Reforma Educativa del Perú que generó en la década de los 70' el gran cambio de nuestro sistema educativo, gestor de la Orientación y Bienestar del Educando (OBE) como actividad básica de la educación, méritos que lo convierte en el referente obligado para los especialistas en la temática del Currículo.

Es oportunidad propicia para expresar el reconocimiento público al gran maestro que alentó las ideas sobre la problemática educativa y laboral del país, nutriéndolas en su sabia perspectiva del desarrollo humano, que se ha convertido en una doctrina vital y que de manera virtual se percibe en cada obra de su fructífera y magistral producción intelectual.

En el sentir de los educadores del país y del extranjero, el 28 de octubre, última salida del Señor de los Milagros, día de su fallecimiento, será también una fecha registrada como memorable entre los creyentes de la formación integral de los profesionales, basada en lo cognoscitivo y en lo no cognoscitivo, y en la trascendental práctica de los principios y valores humanos, "terreno poco transitado por los centros educativos y particularmente por las instituciones de educación superior".

\section{PRESENTACIÓN}

Los trabajos que demanda la estructuración del Currículo para una carrera profesional por crear o por revisar, generalmente, se produce por iniciativa de las autoridades con un grupo pequeño de docentes, o con especialistas externos expertos en tecnología educativa, que puede ser desarrollada con diferente propósitos y alcance

Por un lado, esta situación muchas veces se ha convertido en un trabajo más para la gestión de la organización universitaria o facultativa, dinámica que deviene simplemente en un trabajo de actualización o reorganización del Plan de Estudios o del propio Currículo.

Por otro lado, una alta relevancia que se imprima a la toma de decisión por las autoridades para la revisión del currículo, se estaría en el caso de su reestructuración o reforma, lo que en sí representa un cambio organizacional en la gestión universitaria. 
El propósito que persigue este tipo de cambio organizacional resulta ser trascendental y diferente ante otros cambios organizacionales causados por condiciones específicas de orden económico o del mercado, o por clima organizacional, los que pueden ser atendidos por programas específicos orientados a la productividad, competitividad o cultura organizacional.

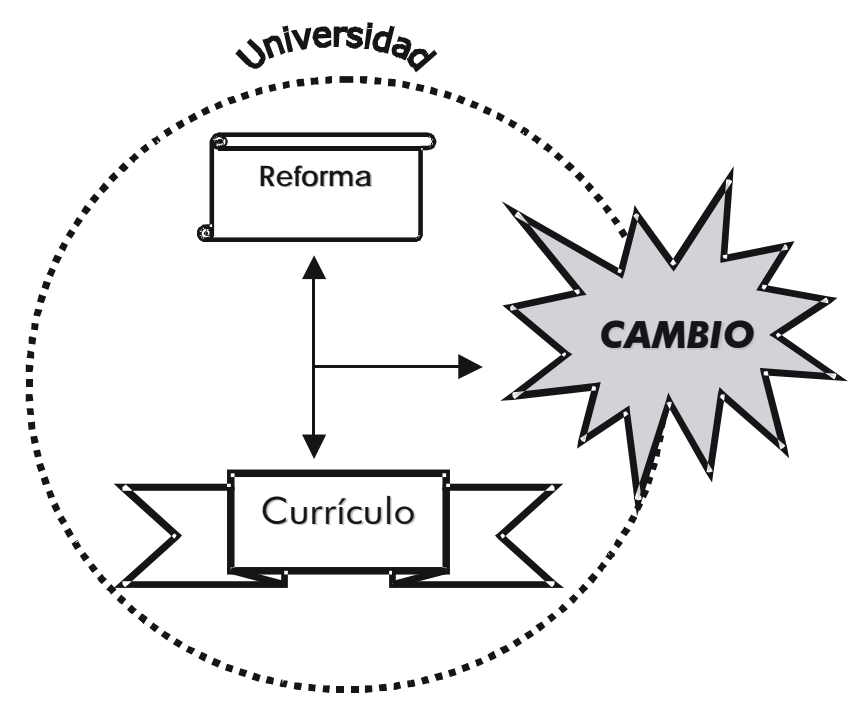

Estamos ante un cambio organizacional a través del Currículo por el cual la Universidad y sus órganos facultativos deberán responder a las exigencias de los cambios que vienen realizando la administración de las organizaciones gubernamentales o privadas para el desarrollo regional y local del país; o de los cambios en el contexto continental y mundial.

Este tipo de cambio organizacional es general, es un cambio profundo de la gestión por reformar o reestructurar su sistema curricular para la formación de profesionales, en este caso particular de la carrera de Psicología.

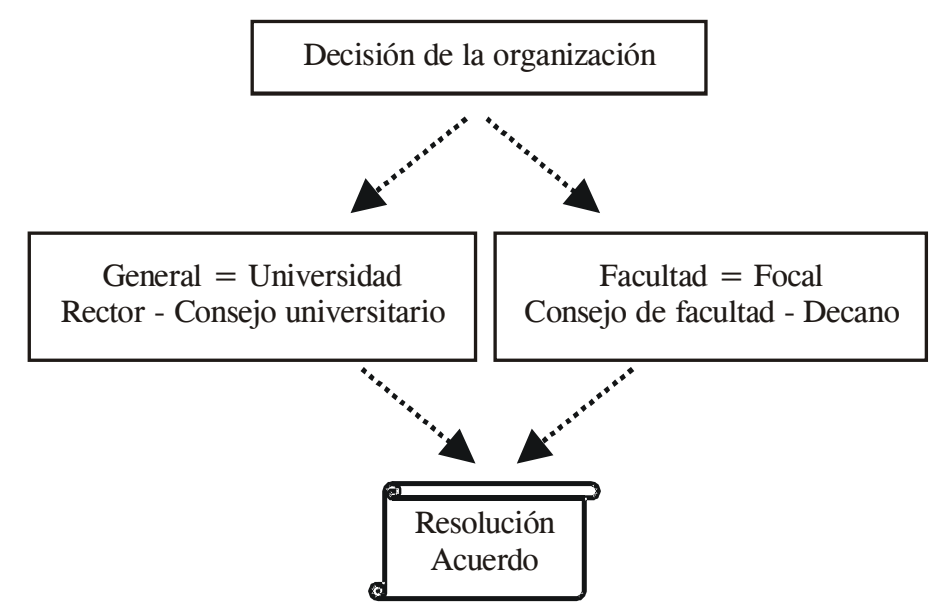


El cambio organizacional por reforma curricular es, en sí misma, la propuesta institucional en su responsabilidad disciplinar para atender las necesidades de nuestra realidad, realidad que actualmente es tan contradictoria entre su crecimiento económico y la extrema problemática social, cultural y política que atraviesa el país y para hacer frente al desarrollo expansivo de los países vecinos y de los bloques continentales.

\section{SISTEMA CURRICULAR}

En la acepción a que un Sistema Curricular es la estructura lograda por la elaboración de un Currículo para la formación de profesionales, deberá ser considerado como un conjunto organizado de elementos o partes interactuantes e interdependientes que se relacionan formando un todo unitario y complejo, que facilite a la organización facultativa a planificar, ejecutar y supervisar todos los procesos educativos plasmados en la concepción del modelo del currículo elegido.

De esta manera, el currículo se convierte en el medio para lograr exitosamente los objetivos determinados por la finalidad y políticas de la Universidad, las que se asumirán como la doctrina institucional para el Cambio Organizacional.

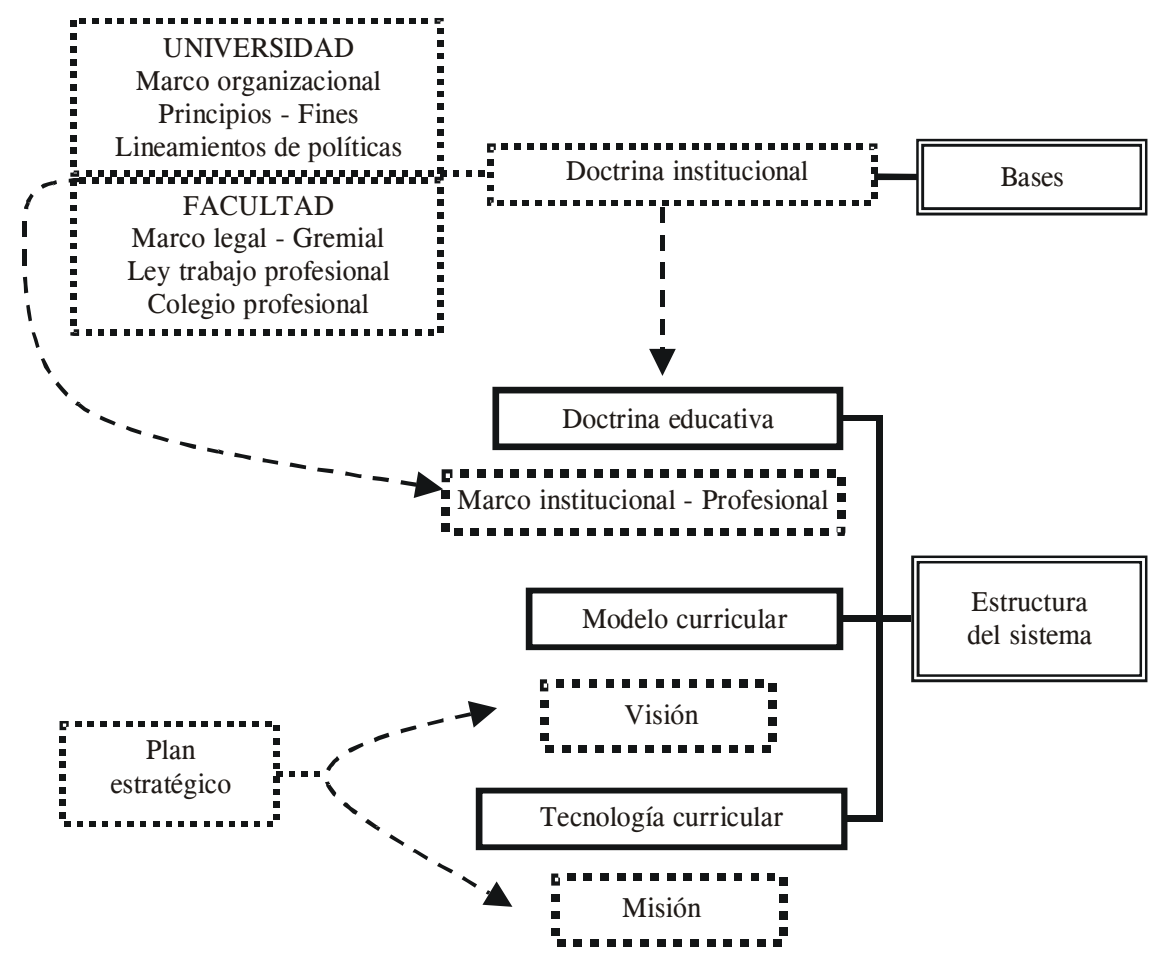


A nivel disciplinar, estaríamos tratando de un sistema curricular para la carrera de Psicología, en el ámbito universitario, cuyo trabajo inteligente y estratégico deberá resultar de la participación de los miembros de la comunidad facultativa, que compartiendo la Visión institucional, asumen el compromiso de la misión de formar profesionales eficientes que entiendan, comprendan los problemas de la sociedad y se identifiquen con los principios y valores humanos.

\section{ENFOQUE CURRICULAR}

Para la formación de los estudiantes de pregrado de la carrera profesional, se requiere de un currículo que, en forma explícita y operativa, sea la respuesta efectiva que genere:

- La atención de las necesidades que exige nuestra realidad social, cultural y el avance científico y tecnológico;

- Que permita hacer frente a los requerimientos de las organizaciones productivas de bienes y servicios, a las políticas de desarrollo del país, y a los efectos de la globalización;.

- Que cambie el tradicional discurso político, coadyuvando de esta manera, decididamente, al auténtico desarrollo sostenible que propicia el establecimiento del punto de equilibrio entre el desarrollo económico y las necesidades de la gente, por un lado, y al mantenimiento de un medio ambiente sano, por otro lado.

El Currículo, siendo el enlace entre el mundo de la concepción y el mundo de la realidad, tendrá como propósito, brindar una formación integral, de naturaleza humanística y científica.

El currículo reformado o reestructurado implica no sólo cambio para un mayor trabajo universitario, significa el cambio a un enfoque diferente de ?ver y comprender el currículo, no como un conjunto de componentes cognoscitivos (las diversas ciencias que se acostumbra impartir en cada carrera), sino como un conjunto de componentes cognoscitivos y también no cognoscitivos?

El Currículo, en una perspectiva holística, deberá integrar la investigación, la docencia y la proyección universitaria y que sistemáticamente deberá tener coherencia interna y una coherencia externa con nuestra realidad local, regional y / o nacional.

El Currículo propiciará a que la gestión de la organización facultativa mantenga permanentemente una interrelación con los sistemas de evaluación interna, y externa con el ámbito universitario a nivel nacional e internacional con respecto a la carrera profesional de Psicología.

El Currículo, para su administración, deberá contar con el apoyo de sus autoridades facultativas, quienes deberán desarrollar lo máximo de sus capacidades profesionales para que su gestión logre satisfacer las expectativas de los estudiantes (usuarios), facilite la realización profesional de los docentes (operadores), y sobre todo que sea un medio coadyuvador en la atención de las demandas sociales del país.

Para comprender el alcance y profundidad del cambio organizacional mediante una reforma curricular, deberán precisarse previamente los niveles de significancia del análisis y debate 
de las concepciones que determinarán el modelo del Currículo deseado, particularmente, para la formación profesional del Psicólogo.

Por tanto, el currículo en su etapa de diseño, ejecutará en un primer nivel de trabajo, el debate y consenso de las Concepciones "trascendentales para la profesión del psicólogo", que corresponden a las concepciones del ser humano, de la educación y, por la dinámica profesional del psicólogo, deberá estar presente en este nivel, la Concepción de la Salud, que generalmente no es tomada en cuenta, no obstante su importancia que representa para los psicólogos, que es una de las razones del propósito y objetivo de la ponencia en el presente simposio.

En un segundo nivel de trabajo, están las concepciones de la Psicología como ciencia y como profesión; y la estratégica concepción del Currículo.

En el tercer nivel de trabajo, se efectuará el análisis y debate de las concepciones del aprendizaje, de la enseñanza y de la evaluación.

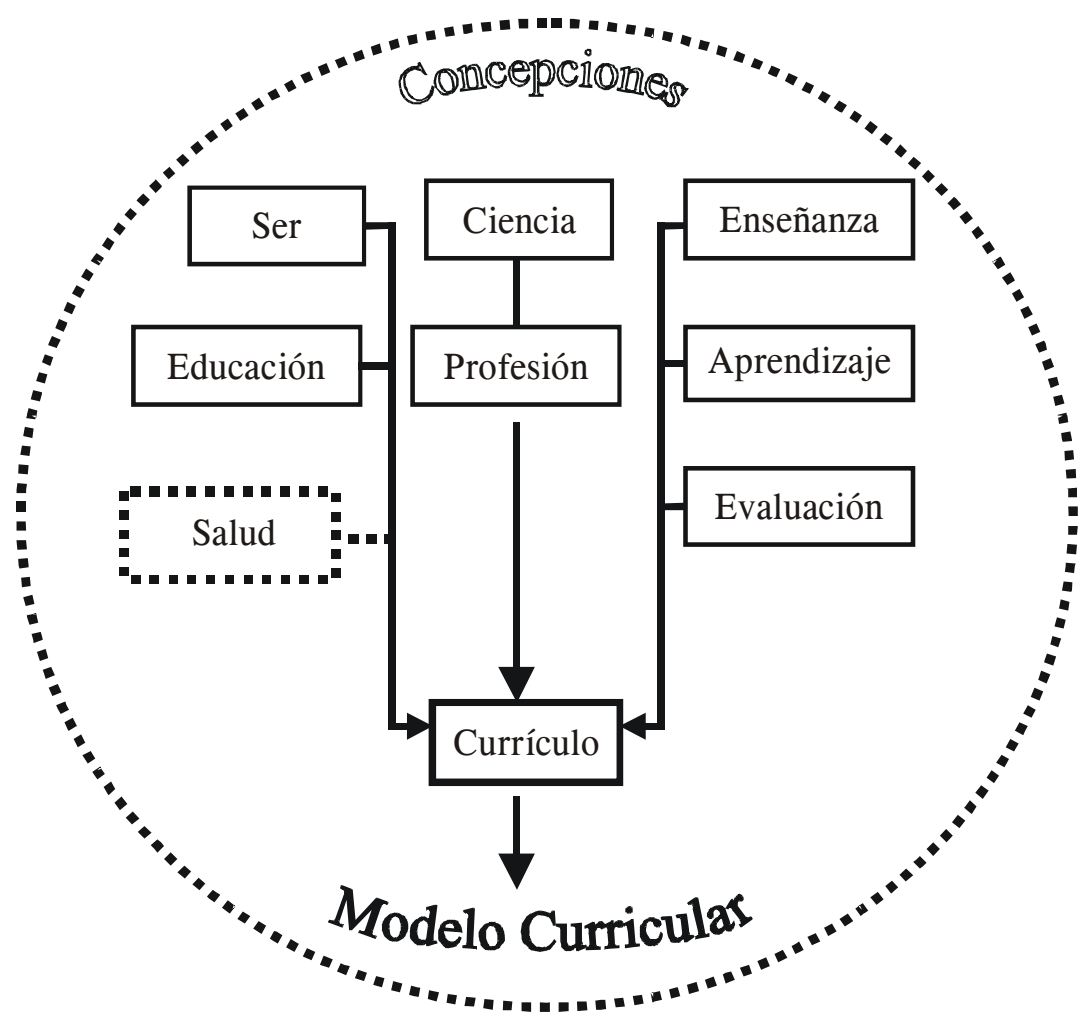

Reforma curricular - Cambio organizacional - Cambio de la manera de pensar.

A un nivel sectorial, lo relevante de incluir la concepción de la salud en el diseño curricular para la formación de los profesionales de la salud, es que brindará aportes significativos a las organizaciones del país en los diferentes niveles de gestión para el cambio profundo de la tradicional manera de pensar con respecto a la salud, y ello permitirá: 
1. Establecer la concordancia del ejercicio profesional con la normatividad laboral y gremial para cada sector del colectivo de los Profesionales de la Salud.

2. Honrar nuestro compromiso profesional con el ser humano y su salud, con el ser humano y su desarrollo integral, es decir, hacer lo que debe hacerse para que el ser humano logre alcanzar su bienestar biopsicosocial.

3. Establecer coherencia e interrelación funcional en el contexto laboral del colectivo de los Profesionales de la Salud, entre los cuales se encuentran los psicólogos (clínicos, educativos, sociales, organizacionales, etc.) para el logro exitoso de los objetivos de las Políticas de Salud.

4. De lograrse este propósito, será un aporte para que el Estado pueda realizar un cambio profundo en la redefinición de la Política de Salud, esto implica que los gobiernos de turno deberán cambiar la tradicional formulación de sus políticas de salud sobre la base de la convocatoria de exclusividad con los profesionales cuya praxis esta orientada a curar la enfermedad (malestar), para pasar hacia una nueva perspectiva de convocatoria de inclusividad con el conjunto de los profesionales de la salud constructores y operadores de la salud (bienestar).

De esta manera, se logrará la unión de los esfuerzos del colectivo de los Profesionales de la Salud de los que curan las enfermedades, como de los que previenen o evitan la enfermedad y de los que promocionan la salud, en una misión de compromiso de conjunto para beneficio de nuestra sociedad y del país en general.

\section{MODELO CURRICULAR}

Asumiendo lo expuesto sobre la naturaleza, características y las concepciones para la estructuración del Currículo con la previa aplicación del criterio mutatis mutandis, se considere como recomendable el optar por el modelo del Currículo Integral, por ser el más indicado en la formación de los estudiantes de pregrado de la carrera profesional de Psicología, como futuros integrante del colectivo de los Profesionales de la Salud.

La administración del modelo de Currículo Integral, deberá enfocarse con criterio prospectivo, con una orientación hacia la calidad académica y acreditación, y con un Plan de estudios estructurado en áreas de formación general, formación profesional básica, formación especializada, investigación, prácticas preprofesionales y actividades formativas.

La importancia que impriman los responsables de la organización facultativa en la gestión del Currículo de considerar estos criterios, estarán también coadyuvando a los esfuerzos de la universidad en su trabajo interno hacia la calidad y excelencia, y al trabajo externo que viene desarrollando la Asamblea Nacional de Rectores en la implementación de los estándares de la autoevaluación para los fines de acreditación.

En resumen, el cambio organizacional, a través de una reestructuración o reforma curricular, deberá impulsar y lograr el establecimiento de una doctrina Institucional basada en la finalidad y políticas de la Universidad, de una Visión Institucional compartida, de una Misión de compromiso entre todos los miembros del órgano facultativo, de potenciar los productos que se obtienen en la aplicación necesaria y previa de un Plan Estratégico. 
De esta manera, el cambio será profundo, resultado de un trabajo participativo por consensus omnium, y que para lograr exitosamente sus objetivos dependerá en gran parte del apoyo permanente de la organización universitaria, como un todo.

Por tanto, resulta imprescindible que la formación profesional de la carrera de Psicología en el ámbito universitario, deberá estar enmarcada en la perspectiva de un Currículo Integral para una gestión sistamática que imprima la dinámica dentro de los principios de coherencia interna y externa de ésta, siendo la alternativa objetiva y saludable, permitirá asumir conscientemente la responsabilidad a que los egresados de la Universidad como Profesionales de la Salud, son la propuesta institucional inteligente y estratégica que coadyuvará al desarrollo sostenible de nuestro país.
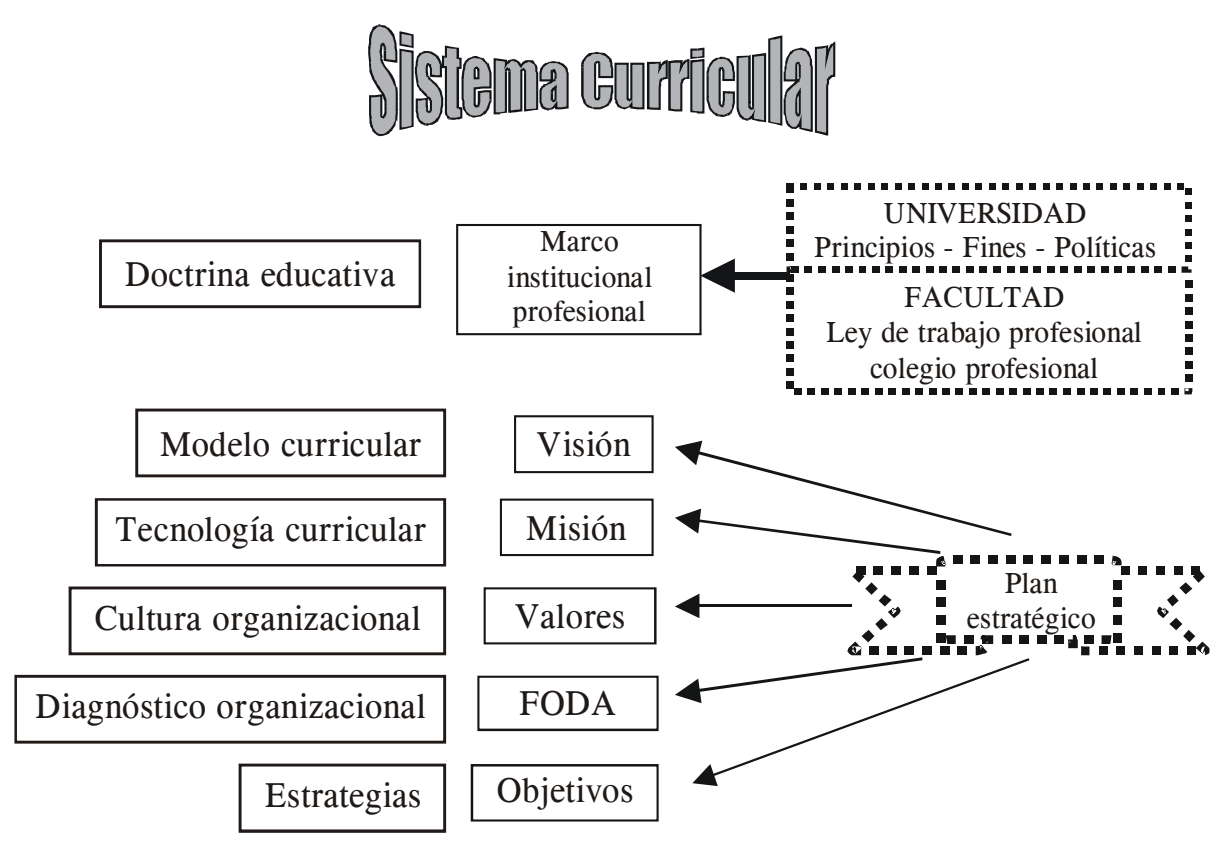

\section{REFERENCIAS BIBLIOGRÁFICAS}

1. Datum International. (2001).La encuesta del Milenio: Delincuencia. Publicado El Comercio 2000.

2. Deming, Edward. (1990) The Deming Approach.

3. Escuela Académico Profesional de Psicología. (2005_May). Currículo Reformado. UNMSM.

4. ONU. (2000). La ciencia en la UNESCO. Publicación de CONCYTEC. 
5. Orellana, O. (1998-2004-2005). Seminarios Curriculares de la Facultad de Psicología. UNMSM.

6. Peñaloza., W. (2000). El Currículo Integral. Editores Optimice.

7. Peñaloza., W. (2003). Los propósitos de la Educación. Fondo Editorial Pedagógico San Marcos.

8. Perea, J L. (2005-May). Reforma Curricular. Clase abierta: Cambio Organizacional. UNMSM.

9. Perea, J L. (2005-Jun). Propuesta para la Reforma Curricular-Sección Organizacional. UNMSM

10. Perea, J L. (2005-Jul). Reforma Curricular de la EAP. Análisis cualitativo-cuantitativo. UNMSM.

11. Perea, J L. (2005-Oct). Currículo y Cambio Organizacional. Ponencia Congreso Internacional de Psicología. Lima-Perú. UNMSM.

12. Puente, R E. (2005). Reestructuración del Currículo. Tesis Doctoral.. UPIGV.

13. Senge, P. (1999). La Danza del Cambio. Editorial Norma.

14. UNESCO. (1998). Rendimiento escolar en América Latina. Publicado el 2002 en El Peruano. 